\title{
Sublimation of a Vibrated Granular Monolayer: Coexistence of Gas and Solid
}

\author{
Andreas Götzendorfer, ${ }^{1, *}$ Jennifer Kreft, ${ }^{1,2}$ Christof A. Kruelle, ${ }^{1}$ and Ingo Rehberg ${ }^{1}$ \\ ${ }^{1}$ Experimentalphysik V, Universität Bayreuth, D-95440 Bayreuth, Germany \\ ${ }^{2}$ Center for Nonlinear Dynamics and Department of Physics, University of Texas, Austin, TX 78712, USA
}

(Received 18 April 2005; published 23 September 2005)

\begin{abstract}
The fluidization of a monolayer of glass beads in a horizontally and vertically vibrated annular container is studied. At peak forcing accelerations between 1.1 and $1.5 \mathrm{~g}$, a solidlike and a gaslike domain coexist. The solid fraction decreases with increasing acceleration and shows hysteresis. The sharp boundaries between the two regions travel around the channel faster than the particles are transported. Complementary to our experimental studies, a molecular dynamics simulation is used to extract local granular temperature and number density. It is found that the number density in the solid phase is several times that in the gas, while the temperature is orders of magnitude lower.
\end{abstract}

PACS numbers: 64.70.Hz, 05.70.Ln, 05.70.Fh, 81.05.Rm

Dry ice (solid $\mathrm{CO}_{2}$ ) at atmospheric pressure provides an example of the phenomenon called sublimation, i.e., the direct transition of a substance from the solid to the gaseous state. In nature this phenomenon is observed in the dry valleys at the margins of Antarctica [1], and technologically exploited for the production of instant coffee by freeze-drying. Here we present a quantitative characterization of sublimation occurring as a nonequilibrium phase transition in a granular system.

Granular material, i.e., an ensemble of macroscopic cohesionless particles, undergoes phase transitions between solidlike, liquidlike, and gaslike states [2]. Since collisions between grains are inelastic, energy must be constantly fed into the system in order to maintain a fluid phase; i.e., a granular liquid or gas is always away from equilibrium.

The coexistence of different phases is known to accompany the fluidization of granular material. For example, submitting a two-dimensional vertical packing of beads to vertical or horizontal vibrations fluidizes the upper layers, while the lower lying material still maintains a crystalline structure [3-5]. Likewise, investigations into the fluidization of a vertically vibrated horizontal submonolayer demonstrated that domains of different phases can coexist [69]. Coarse graining of the coexisting phases has been quantitatively characterized in electrostatically driven granular material $[10,11]$. Other examples for driven systems where the equipartition of kinetic energy is violated are bidisperse mixtures [12-14] and monodisperse systems in a container separated into two compartments by a wall $[15,16]$. The latter experiment became popular as the granular "Maxwell's demon" [17].

In this Letter, we study a monodisperse system where equipartition of energy is violated by coexisting gaseous and solid domains, even though particle motion is fully three-dimensional and not restricted by guiding partitions. In a specially designed apparatus we made the solid phase rotate to demonstrate that the coexistence of solid and fluid regions is not caused by small potential inhomogeneities in the forcing, particle container interactions, or a tilt of the apparatus.

Our container consisted of a $2 \mathrm{~cm}$ wide annular channel with open top, $7 \mathrm{~cm}$ high Plexiglas walls, and a radius of $R=22.5 \mathrm{~cm}$ giving a circumference of $L_{0}=141 \mathrm{~cm}$ (see Fig. 1). Using grounded stainless steel as floor material and working at elevated relative humidity $(>50 \%)$ kept electrostatic charges negligible and led to reproducible results.

The annular channel is suspended with elastic bands in a frame with four symmetrically arranged rotating vibrators firmly connected to the channel $[18,19]$. We used unbalanced-mass vibrators that are well established in industrial applications. Their working principle is based on the joint action of two equal unbalanced masses rotating in opposite directions, thereby exciting a linear sinusoidal vibration. The four driving units are coupled to the motor via a common central gear box, which keeps all drives in the same phase. The driving torque is transmitted from the gear box to the vibrators by use of rotating rods connected with compensation clutches.

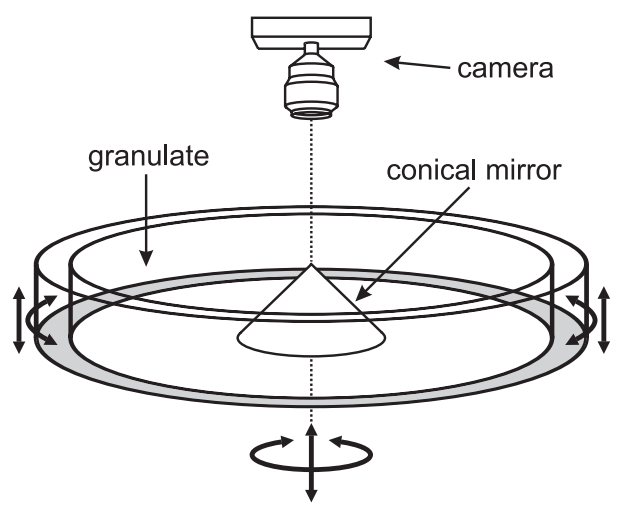

FIG. 1. Geometry of the container and imaging system. Directions of basic vertical and rotational oscillations are indicated by arrows. For a description of the driving system see text. 
The granular material was thus agitated by vertical sinusoidal oscillations of the container $z(t)=$ $A_{\mathrm{v}} \sin (2 \pi f t)$. Additionally the ring performed a sinusoidal angular oscillation $\varphi(t)=\left(A_{\mathrm{h}} / R\right) \sin (2 \pi f t+\pi / 2)$ at the same frequency $f$ around its axis of symmetry. With the mounted unbalanced masses the amplitude in the vertical direction, $A_{\mathrm{v}}$, decreased slowly with frequency from 2.27 to $2.22 \mathrm{~mm}$. The horizontal amplitude, $A_{\mathrm{h}}$, in the center of the channel was $93.4 \% \pm 0.5 \%$ of the vertical amplitude. Accordingly, every point of the channel floor followed an almost circular trajectory in a plane tangent to the ring. For the experiments a densely packed monolayer of spherical basalt glass beads (density $\rho=3.0 \mathrm{~g} / \mathrm{cm}^{3}$ ) with diameter $d=1.18 \pm 0.03 \mathrm{~mm}$ was prepared on the channel floor. Its mass corresponded to 21500 particles.

The granular system was observed from the top via a conical mirror placed in the center of the ring, similar to Ref. [20]. Thus a side view of the whole channel was captured with a single high-speed digital camera (resolution: $1280 \times 1024$ pixels at rates up to 500 images per second). Digital image processing delivers $360^{\circ}$ panoramic side views of the granular profile in the channel as presented in Fig. 2. One image per forcing cycle was recorded at a fixed phase using an electro-optical triggering system. The channel was lit from outside through diffusive parchment paper wrapped around the outer wall; hence particles appear dark in front of a bright background.

To have access to the positions and velocities of all particles, experiments were replicated using a threedimensional molecular dynamics simulation applying an event-driven algorithm described in Ref. [21]. When not mentioned explicitly, the length of the system $L_{0}$ and the number of particles was one-quarter of those in the experiment to decrease the calculation time. The entire channel moved both vertically and horizontally with the same values of $f, A_{\mathrm{v}}$, and $A_{\mathrm{h}}$ as in the experiment. The parameters characterizing changes in relative surface velocity for both ball-ball and ball-wall collisions were the coefficient of sliding friction $\mu=0.5$ and the maximum rotational coefficient of restitution $\beta_{0}=0.35$. These values were chosen because they successfully reproduced surface wave patterns observed in vertically vibrated granular media [21]. To match the minimum layer depth required for surface wave formation in experiment, the coefficient of normal restitution for interactions between particles, $e$, was set to 0.97 . The coefficient of restitution with the walls was the only fit parameter and a value of 0.93 was found to quantitatively reproduce the experimental results. To prevent inelastic collapse, the coefficient of normal restitution for both types of collisions approaches unity as the normal velocity $v_{\mathrm{n}}$ approaches zero according to $\max [e, 1-(1-$ $\left.e)\left(v_{\mathrm{n}} / \sqrt{g} \bar{d}\right)^{3 / 4}\right][21]$.

If the granulate is driven at peak dimensionless accelerations $\Gamma=(2 \pi f)^{2} A_{\mathrm{v}} / g$ up to 1.1 the grains spread homogeneously in the channel. In close-up high-speed movies the whole monolayer looks like a single completely inelastic object. The particles basically maintain their relative positions. Above $\Gamma=1.5$ the granular material is fully fluidized and resembles a gas [22]. Particles jump some centimeters (several tens of particle diameters) high, and their motion seems to be random. At intermediate forcing $(1.20<\Gamma<1.52)$ both phases can coexist as demonstrated in Fig. 2. Starting from a homogeneous, solid monolayer fluidized regions first appear at $\Gamma=1.28$. In contrast, arriving from a completely fluidized state density fluctuations lead to gas collapse and the formation of solid regions at $\Gamma=1.40$. At $\Gamma$ values between the spontaneous creation of one of the two phases and the limits of the coexistence region, the system is hysteretic. The generation of coexisting phases usually gives rise to several separated solid and fluid domains. Condensed regions merge to form a single solid domain surrounded by gaseous granulate after approximately $10 \mathrm{~min}$. Thenceforward, this single solid domain stays intact, preserving its dimensions. The phase boundary is remarkably sharp and is always small compared to the size of the solid and gas phases. The boundaries between the solid and fluid regions travel along the
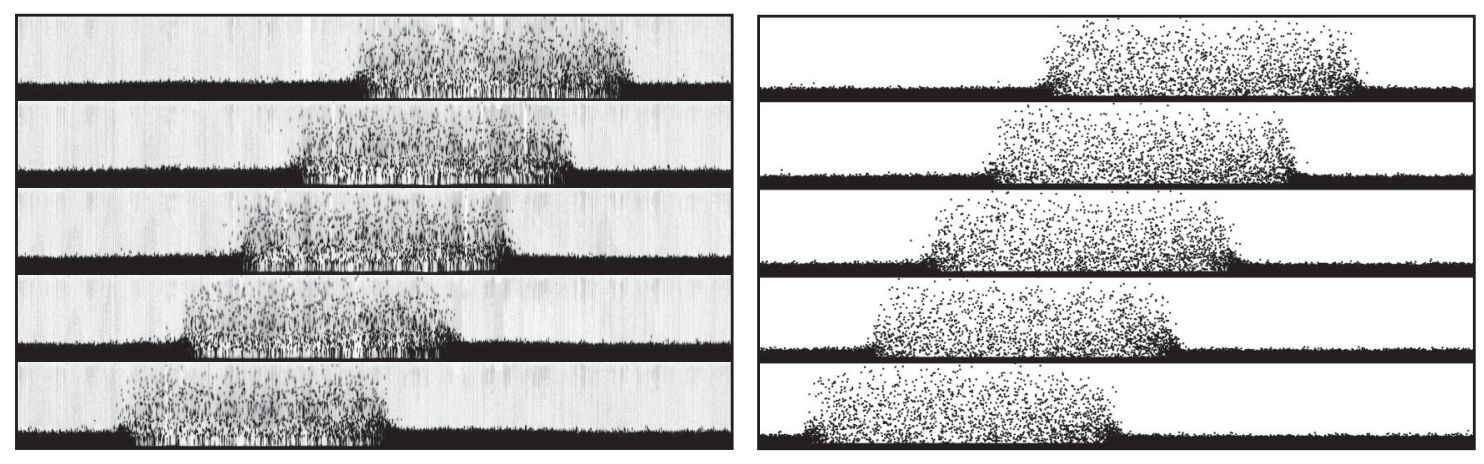

FIG. 2. Snapshots through the inner side wall of the channel covering $360^{\circ}$ from experiment (left) and simulation (right) taken at $z(t)=0$ during the downwards motion of the container. Here the system sizes of simulation and experiment are equal. Time increases from top to bottom by $1.72 \mathrm{~s} \mathrm{(20} \mathrm{cycles)} \mathrm{between} \mathrm{consecutive} \mathrm{snapshots.} \mathrm{For} \mathrm{clarity} \mathrm{all} \mathrm{images} \mathrm{are} \mathrm{stretched} \mathrm{in} \mathrm{the} \mathrm{vertical} \mathrm{direction} \mathrm{by}$ a factor of $4 .(f=11.6 \mathrm{~Hz}, \Gamma=1.23$. $)$ 
channel due to the presence of a horizontal oscillation component inducing material transport $[18,19]$.

For the determination of the solid fraction, i.e., the length of the solid region divided by the circumference of the channel, $L_{s} / L_{0}$, and the velocity of the phase boundaries, we use side views as shown in Fig. 2. As an order parameter for the two states we define the density difference $\Delta n$ between a lower and an upper region in the container. The lower region ranges from the container floor to the surface of the solid phase (obtained from the maximum density gradient), while the top region extends from there to a height of $30 \mathrm{~d}$ (corresponding to the height shown in Fig. 2). A measure for the density is determined from the average gray value in experiment or the number density in simulation. In the solid phase $\Delta n$ will be high while in the gas phase a low value will result. This order parameter, coded in gray scale, is plotted as a function of horizontal position $x$ and time in the inset of Fig. 3. The phase boundaries are determined from the largest gradients in these plots after short wavelength fluctuations have been filtered out. The lengths of the condensed phase and the velocities of the phase boundaries are constant once the coarsening process is over.

The length of the solid phase $L_{s}$ shown in Fig. 3 declines sharply from complete coverage to about half the circumference within the lower hysteretic region. It follows a more gentle virtually linear decrease for the rest of the range of coexistence. Simulation and experiment agree very well in the nonhysteretic part. In this region simulation results for the relative length of the condensed phase do not change when the system is 4 times longer. The deviations in the hysteretic regions, however, are partly a consequence of the shorter channel length in the simulations.

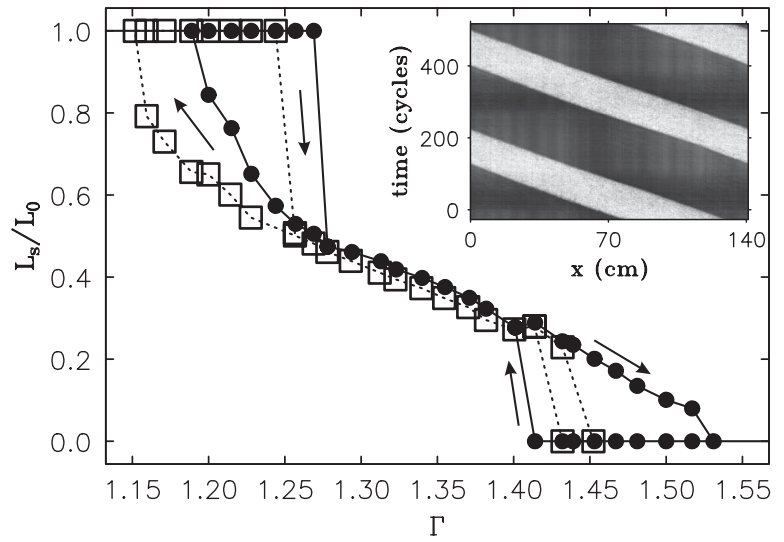

FIG. 3. Solid fraction $L_{s} / L_{0}$ as a function of the peak container acceleration $\Gamma$ in experiment (filled circles) and simulation (open squares). Arrows indicate how the system evolves in the hysteresis loops. The inset is a space-time diagram of $\Delta n$ (see text for definition) from experiment for the parameters of Fig. 2. Solid regions appear dark.
In Fig. 4 the dimensionless velocity $v /\left(2 \pi f A_{v}\right)$ of the phase boundary is given as a function of $\Gamma$. It exhibits an approximately linear increase in the range of coexistence. Additionally the mean transport velocity was calculated from the particle displacements in the simulation over 50 forcing cycles. Its decline with increasing $\Gamma$ is a consequence of the particles in the gas phase being transported less efficiently than those in the solid phase, as observed in experiment. The difference in transport velocity for the two phases also implies a continuous deposition at the front of the condensed phase and sublimation at the end. This cyclic process of solidification and fluidization causes the gap between the mean transport velocity and that of the phase boundary.

For the numerical investigation of the granular temperature and number density, the simulations were started from the circularly forced case after all transients have died out. It turned out that the coexistence remains stable after switching off the horizontal forcing. The channel was subdivided into thin vertical slices and the number of particles in each of them counted and normalized by the average value $n_{0}$. The normalized granular temperature $T$ is defined here as the mean square horizontal velocity per slice divided by gravity and particle diameter, $\left\langle v_{\mathrm{hor}}^{2}\right\rangle / g d$. Figure 5 contains a showcase result featuring a gas phase with a granular temperature 2 orders of magnitude higher than in the solid, while its number density is a factor of 3.5 lower.

In conclusion, our system allows a quantitative characterization of sublimation and the coexistence of gas and solid phases in granular matter. This is due to the additional horizontal oscillation component that makes the pattern rotate, and thereby demonstrates that the coexistence of solid and fluid regions is not caused by small potential inhomogeneities in the forcing, particle container interactions, or a tilt of the apparatus. The horizontal forcing also

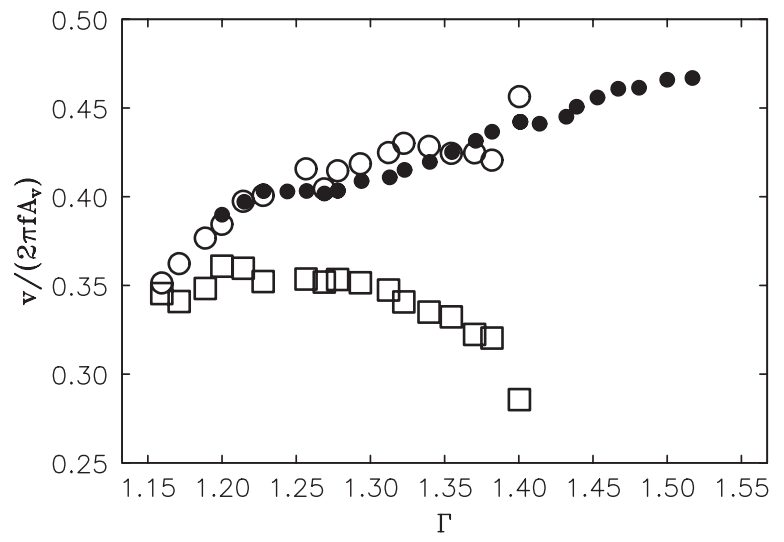

FIG. 4. Dimensionless velocity $v /\left(2 \pi f A_{v}\right)$ of the phase boundaries as a function of the peak container acceleration $\Gamma$ in experiment (filled circles) and simulation (open circles). Open squares indicate the mean transport velocity obtained from numerical simulations. 


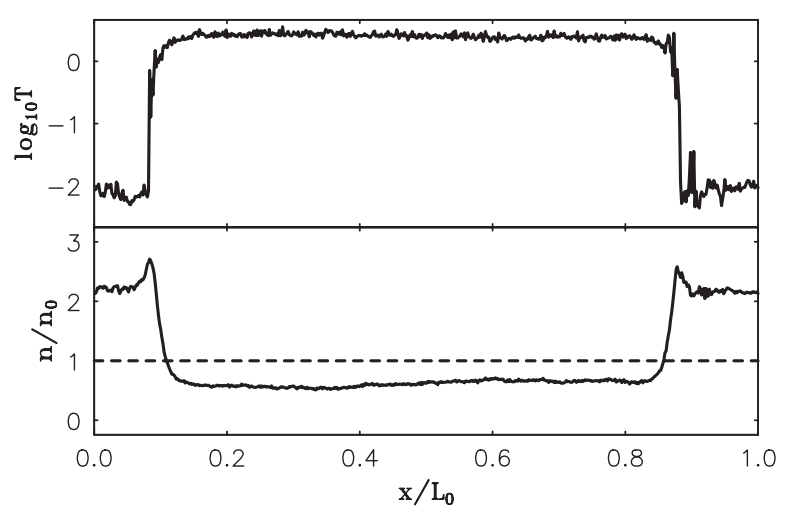

FIG. 5. Dimensionless temperature (top) and number density (bottom) from simulations with purely vertical forcing and $\Gamma=$ $1.40, f=12.53 \mathrm{~Hz}$. The broken line in the lower graph indicates the average number density.

assists the coarsening process because the transport velocity in the solid regions increases slightly with their length. Larger solid regions catch up to smaller ones and incorporate them until all solid regions have merged. This process is irreversible. Finally, a unique time independent state with constant length of the solid phase and constant velocities of the phase boundaries is established.

The simulations show that the number density in the solid phase is several times that in the gas, while the granular temperature is 2 orders of magnitude lower. That ratio is less extreme for the vertical component of the kinetic energy, namely, only about a factor of 2 . Such a lack of equipartition of energy is allowed in nonequilibrium systems.

An open question remains why and how a constant solid fraction is established. We believe that part of the answer can be extracted from numerical simulations as presented in Fig. 5. Solid particles are excited by the plate and will diffuse towards the more dilute gas phase at the boundary. In the steady state this flux is balanced by the uppermost gas particles entering the region of the solid phase through ballistic jumps. If the solid phase shrinks due to fluctuations, the gas density must increase because the solid phase has a higher number density. This leads to an increase in the back flux of particles from the gas towards the solid, while the diffusion from the solid to the gas phase remains constant. Therefore the solid state expands again, thus stabilizing the steady state solid fraction.

While in equilibrium phase transitions minimization of free energy determines the relative amounts of gas and solid phases in contact with a common heat bath, there is no recognized free energy for granular materials as pointed out by Prevost and co-workers [9]. Future detailed studies of this system might offer insight into what plays the role of free energy here. The nature of the coexistence of gas and solid in shaken granular matter with a self-stabilizing discontinuous jump of density and temperature across the boundary remains an intriguing phenomenon.

We thank Matthias Schröter for illuminating discussions and acknowledge the support of Deutsche Forschungsgemeinschaft for funding the presented research work as part of the program "Verhalten granularer Medien."

*Electronic_address: andreas.goetzendorfer@unibayreuth.de

[1] I. Hawes, A.-M. Schwarz, D. Sutherland, and C. HowardWilliams, Water Atmosphere 11, 16 (2003).

[2] H. M. Jaeger and S. R. Nagel, Rev. Mod. Phys. 68, 1259 (1996).

[3] E. Clément and J. Rajchenbach, Europhys. Lett. 16, 133 (1991).

[4] J. A.C. Gallas, H. J. Herrmann, and S. Sokołowski, Physica (Amsterdam) 189A, 437 (1992).

[5] G. H. Ristow, G. Straßburger, and I. Rehberg, Phys. Rev. Lett. 79, 833 (1997).

[6] J. S. Olafsen and J. S. Urbach, Phys. Rev. Lett. 81, 4369 (1998).

[7] W. Losert, D. G. W. Cooper, and J. P. Gollub, Phys. Rev. E 59, 5855 (1999).

[8] X. Nie, E. Ben-Naim, and S. Y. Chen, Europhys. Lett. 51, 679 (2000).

[9] A. Prevost, P. Melby, D. A. Egolf, and J. S. Urbach, Phys. Rev. E 70, 050301(R) (2004).

[10] I. S. Aranson, D. Blair, V. A. Kalatsky, G. W. Crabtree, W.-K. Kwok, V. M. Vinokur, and U. Welp, Phys. Rev. Lett. 84, 3306 (2000).

[11] M. V. Sapozhnikov, I. S. Aranson, and J. S. Olafsen, Phys. Rev. E 67, 010302(R) (2003).

[12] R. D. Wildman and D. J. Parker, Phys. Rev. Lett. 88, 064301 (2002).

[13] K. Feitosa and N. Menon, Phys. Rev. Lett. 88, 198301 (2002).

[14] A. Barrat and E. Trizac, Granular Matter 4, 57 (2002).

[15] H.J. Schlichting and V. Nordmeier, Math. Naturwiss. Unterr. 49, 323 (1996).

[16] K. van der Weele, M. van der Meer, M. Versluis, and D. Lohse, Europhys. Lett. 53, 328 (2001).

[17] J. Eggers, Phys. Rev. Lett. 83, 5322 (1999).

[18] R. Grochowski, P. Walzel, M. Rouijaa, C. A. Kruelle, and I. Rehberg, Appl. Phys. Lett. 84, 1019 (2004).

[19] M. Rouijaa, C. A. Kruelle, I. Rehberg, R. Grochowski, and P. Walzel, Chem. Eng. Technol. 28, 41 (2005).

[20] E. van Doorn and R. P. Behringer, in Powders \& Grains 97, edited by R. P. Behringer and J. T. Jenkins (Balkema, Rotterdam, 1997), pp. 397-400.

[21] C. Bizon, M. D. Shattuck, J. B. Swift, W. D. McCormick, and H. L. Swinney, Phys. Rev. Lett. 80, 57 (1998).

[22] Granular Gases, edited by T. Pöschel and S. Luding (Springer, Berlin, 2001). 